Published in final edited form as:

Hosp Pract (Hosp Ed). 1971 March ; 6(3): 47-60.

\title{
The Current Status of Liver Transplantation
}

\author{
THOMAS E. STARZL \\ Dr. Starzl is professor of surgery, University of Colorado School of Medicine, and chief, Surgical \\ Service, Veterans Administration Hospital, Denver
}

\section{Abstract}

More than thirty patients have now undergone liver transplantation in Denver, some more than once, and survivals of up to two and a half years have been achieved. Through this and other experience it has been learned that graft viability is more critical than histocompatibility matching but that the most important factor in the ultimate outcome is prevention of rejection through vigorous immunosuppressive therapy.

Treatment of terminal liver disease by transplantation was founded on the encouragement and knowledge provided by the steadily improving experience in renal transplantation. The liver, however, is a far more complicated organ, and its derangement leads to vastly more complex physiologic impairments. Liver patients are further handicapped, as are heart patients, by the lack so far of a satisfactory means of artificial support comparable to renal dialysis that could take over the organ's compromised functions during the wait for a suitable donor, or during the critical immediate postoperative period. Live donors, of course, are not feasible, timing is of the essence, and the circumstances for obtaining an optimal homograft are rarely if ever ideal. The transplanted liver must function efficiently practically from the moment of anastomosis or the patient is lost.

Despite these and other formidable difficulties, the past 10 years have furnished enough progress in the laboratory and clinic to let us state that liver transplantation is now a feasible and legitimate, albeit imperfect, form of treatment, one that may in certain cases be considered as a last best hope. Human survivals for up to two and one half years have been achieved. A great deal has been and is being learned - at a pace suggesting that the next phase, when liver transplants will have at least as much a chance as kidney grafts now have, is not far off. Eventually much more than that will need to be accomplished, of course, but rather than engage in speculation it would appear more useful here to record how far we have come and to identify the major hurdles immediately ahead.

When research in liver transplantation was in its early stages, it was hoped that as the liver played a significant role in graft rejections, hepatic homografts might enjoy a better fate than other transplants because presumably the grafted liver would not participate in rejecting itself. The case for this rather mystical view seemed even strengthened by certain experiences with laboratory animals. When immunosuppression in canine recipients was stopped after four months a surprising number of animals continued to thrive, with no signs of rejection or with rejection episodes that waxed or waned remittently. One such dog is still alive, with stable liver function seven years after the transplant. This phenomenon of "graft acceptance" had been noted in dogs with renal transplants, but less frequently.

If the liver thus seemed to be an immunologically more favored organ in dogs, its status in pigs - as observed by Garnier in Paris, Terblanche in Bristol, Calne in Cambridge, and in our own laboratory - was even more noteworthy. In some experiments with pigs not treated with immunosuppressive agents, identifiable homograft rejection did not occur. In other experiments, rejection was indolent and spontaneously reversed. This state of affairs applied 
only to a minority of animals. Nevertheless, these results had to be attributed to some special privilege of the liver, since porcine skin and kidney grafts were regularly rejected in the usual way.

These observations in both dogs and pigs (and now in other animals) invited certain hypotheses in addition to the one stated above that the new liver helped create an internal milieu favorable to itself. There were the possibilities that the liver was inherently less antigenic than other organs, that its relatively great antigenic mass was a beneficial factor, that its enormous regenerative capacity made it less susceptible than other tissues to the effects of chronic rejection, or (Caine) that it possessed or released some special factor promoting the induction of specific immunologic tolerance.

Whatever the explanation, overstatement of the case for the liver's privileged status could Jead to erroneous conclusions about the practical requirements for immunosuppressive therapy following hepatic transplantation in man. At a research level, another danger could stem from the notion that hepatic transplantation, espec ially in the pig, is somehow qualitatively unique: The fallacy of such a contention is obvious from the fact that even in the "easy" pig model the majority of untreated liver recipients die from acute rejection. In dogs and humans, control of hepatic rejection may be difficult or impossible in spite of very heavy immunosuppressive therapy.

It is my opinion that liver homografts differ from other oragns only in the degree of host immunologic response they evoke in all species, including the pig. In this context, two key observations first made with kidneys have been extended to the liver, and there is little doubt that they apply to other tissues as well. The first is the reversibility of rejection, which has been well documented in canine, porcine, and human recipients of hepatic homografts. In patients, reversal usually requires intensification of treatment, but it has sometimes been noted without any change in the preexisting therapy, suggesting that such recoveries had an element of spontaneity. As mentioned earlier, "spontaneous remission" of rejection in the absence of all therapy has occurred both in dogs and pigs, particularly the latter. The reactions of the liver in all three species are undoubtedly expressions of the same phenomenon, differing only quantitatively.

The second observation of overriding practical and theoretical interest concerns what has already been referred to as "graft acceptance." In many of the human kidney recipients treated almost a decade ago, it was shown that a melting away of host resistance to the homograft occurred surprisingly early after transplantation, often following an acute rejection crisis. This was manifested by eventual declines in the doses of immunosuppressive agents necessary to retain stable graft function. In many patients, the level of chronic immunosuppression has proved to be less than that which at the outset failed to prevent the onset of a severe rejection.

It is probable that all treatment could be stopped in some of these human renal recipients whom we have now followed for seven or eight years, but we have not dared to take such a drastic step. However, as described earlier, therapy has been successfully discontinued in dogs after kidney transplantation and even more consistently after liver replacement, indicating that graft acceptance may become complete. In pigs, the barrier of natural host resistance is apparently low enough so that the cycle of hepatic graft acceptance can be completed without any immunosuppression at all. Viewed in this way, the curious pig liver experiments become only a special example of, rather than an exception to, a general principle of transplantation. Recently, Perper has provided evidence to support both this concept and the original idea that there is a slight but limited biologic advantage in transplanting the liver rather than the kidney. Perper showed that a three-day course of 
heterologous antilymphocyte globulin (ALG) treatment (or other short-term therapeutic maneuvers) in pigs permitted long-term acceptance of kidneys in precisely the same way as it does with the liver in the absence of all iatrogenic intervention.

It is indisputable that some element of acceptance of various kinds of grafts occurs often in humans under the appropriate conditions of immunosuppression and that the degree to which this develops is a prime determinant of the long-term prognosis. Unfortunately, the reason for the change in the host-graft relationship is not known. More than one immunologic pathway may be involved. Schwartz and Talmage first called attention to the possibility that the continuous presence of a transplanted organ in a host being treated with immunosuppressive therapy could lead to a selective loss of responsiveness to antigens. The suggestion here is that specific lymphocyte clones, induced to replicate by the graft antigens, are thereby rendered more vulnerable to the killing effect of immunosuppressive agents than is the rest of the lymphocyte population. Inasmuch as the maintenance of such activated cell lines appears to be thymus-dependent even in adult life, at least in some experimental animals, it is reasonable to be curious about the effect of thymectomy as an adjuvant immunosuppressive measure. The results of thymectomy in a series of our human renal transplants were inconclusive; though those with thymic excision did not have better survival or superior renal function, there were fewer and less severe histopathologic abnormalities when the grafts were examined long after transplantation.

At any rate, the concept of specific, differential tolerance through "clone stripping" can partly explain the characteristic cycle of rejection and reversal that occurs after whole organ transplantation both in treated animals and man and in the weak, self-resolving crises in the untreated pig. Moreover, this concept is consistent with the fact that a wide variety of agents that are capable of general immunologic crippling can also provide specificity of action under the stipulated conditions of immunosuppressive treatment during presence of the antigen.

To date, few investigations to establish the presence or absence of classic immunologic tolerance to donor tissue have been carried out in human recipients of chronically functioning renal homografts. It would be interesting to know if skin from these donors would be accepted. One of the reasons why such a test has not been carried out in patients is the potential risk of precipitating an immune reaction that could damage the kidney. Of course, the liver recipient's viable donor tissue is not available for such an experiment even if this were a desirable undertaking.

Fritz Bach has provided evidence that at least some kidney recipients develop true tolerance to their donors. Dr. Bach performed mixed lymphocyte cultures with peripheral blood from a number of our renal recipients and their donors two to four years after transplantation. He was able to demonstrate in some cases that recipient lymphocytes no longer developed blast transformation when exposed to killed donor white cells, al though they reacted vigorously to third-party cells. It is important to note that this was not always the case and that animal experiments with skin transplants similarly showed mixed results.

These ambivalent findings do not disprove tolerance through clone stripping so much as they suggest that at least another mechanism of graft acceptance is involved. One such mechanism, termed enhancement, has been envisioned as a process in which immunoglobulins synthesized by the activated lymphoid tissues return to the target tissue and coat it or protect it in some way that is not yet understood. Antigraft antibodies, selectively capable of being absorbed by the nucleated cells of the original donor, have been detected in patients carrying well-tolerated renal transplants. Extensive immunoglobulin deposition has been demonstrated by immunofluorescence techniques in long-functioning 
kidney homografts, but this finding usually has an adverse connotation rather than a favorable one.

An understanding of the means by which grafts are accepted may hold the key to improvements in therapy. When we come to know more about these mechanisms it may prove possible to arrange the conditions required for graft acceptance in advance of the arrival of the homograft rather than to rely on their development while fighting the battle of rejection. The result might well be the prevention of rejection with far less immunosuppressive crippling of the immune apparatus in the critical postoperative period. In this connection it is heartening to refer to the experiments of Stuart and his colleagues at the University of Chicago. When enhancing antibodies were combined with tolerance induction by donor-specific antigen pretreatment, rat renal transplants functioned 18 months and longer, even in the presence of strong histocompatibility barriers, the absence of immunosuppression, and the retention of immunologic reactivity to most antigens.

Another way to improve clinical results might be by effective donor-recipient matching of histocompatibility ( $\mathrm{L}_{-\mathrm{A}}$ ) antigens. Unfortunately, the state of our knowledge about human histocompatibility systems is still primitive. While a good match between siblings appears to provide a more favorable prognosis after renal transplantation than a poor match, our experience with unrelated subjects provides no such correlation and has led us for the moment to the possibly heretical practice of ignoring the question of $\mathrm{HL}_{\mathrm{A}}$ matching altogether in cadaveric cases. In liver transplantation, in which nonrelated cadaveric sources must be utilized exclusively, we have had some excellent results with poor histocompatibility matches and some discouraging, results despite close matches. Not only has a correlation with tissue typing been absent with regard to clinical outcome but $\mathrm{K}$. A. Porter of London has found no connection between the quality of the match and the appearance of the hepatic homograft at subsequent histologic examination. Until the discrimination of the matching methods is improved in nonrelated cases, we see no justification for denying a patient an available organ solely on the basis of poor serologic histocompatibility. Nor do we even use most favorable matching as an instrument of selection among candidates for transplantation. At the present time, a more valid criterion may be: Who has the most pressing need?

None of this should be construed as denigrating the ultimate value of histocompatibility determination in the transplantation of the future. What is at issue today is acceptance of the fact that typing between nonrelated individuals with serologic methods is imprecise, incomplete, and incapable of consistently predicting the extent of the antigenic confrontation in individual cases or the effectiveness with which immunosuppression may be used. The mixed lymphocyte culture method of Hirschhorn and Bach may be more discriminating and its findings more relevant for the selection of recipients. However, the procedure takes the better part of a week to perform, much too long for practical application in most cadaveric cases at the present time.

Here we turn to the question of logistics, for the problem of obtaining a fresh, functioning, nonischemic liver is paramount and provides the strongest correlation with success or failure. A major advance in one technical aspect of logistics, that of organ preservation and banking, would not only reduce the present inevitable waste of cadaveric organs but would also make more feasible the use of tissue typing when these techniques become more predictive. Selection on the basis of favorable histocompatibility would then become far more realistic and meaningful.

In the meanwhile, the most favorable prognostic thing that can be done for the hepatic transplantation candidate is to assure him an undamaged liver. 
In discussing homograft quality, the technical details of organ preservation become interwoven with, or even distinctly secondary to, ethical considerations about the conditions for the pronouncement of donor death and problems of cooperation by the medical and lay community. Unquestionably, one of the most important advances in transplantation has been social in nature - acceptance by the public of the concept of cadaveric organ removal. In turn, this was made possible by a willingness of many in the medical profession to identify potential donors, approach family members at the time of their bereavement, or indicate in other ways their belief in the propriety of these efforts. The consequence has been a major contribution to our transplantation program by a well-informed community.

I believe that the transplantation team's style of community relations is a vital factor in determining the success of a cadaveric program. The difficult decisions required by the several parties to organ replacement cannot be made objectively in the atmosphere of a fishbowl. I have the impression that securing donors is less of a problem where transplant teams deliberately keep the matter in its proper perspective as a private concern between doctor and patient. The general public, quite naturally, is keenly interested in the drama and medical achievement represented by transplantation, and it is likewise important to transplantation that the public be informed of legitimate progress. This can and has been done in many areas impersonally, with restraint, without exaggeration, and without infringing on the personal right to privacy of the individuals involved.

After the donor has been identified and made available, an effort is made to maintain good liver perfusion up to the last possible moment in order to minimize the ischemic damage that even a short unperfused period may wreak under normothermic conditions. The extraordinary efforts required to prevent circulatory depression in the donor in the face of a hopeless prognosis usually require explanation to relatives and represent a problem of emotional substance to all parties.

Ultimately, a final decision to discontinue supportive measures must be made after all is in readiness to proceed with the recipient. During the first years of liver transplantation at the University of Colorado a considerable physiologic penalty was accepted because of criteria that insisted on both brain death and cessation of heartbeat before organ removal. The price of this insistence was the loss of critical time and the occurrence of ischemic damage during both the agonal stages of circulatory failure and in the minutes after cardiac arrest. The reason for accepting these conditions was the fear that the quality of terminal care for the donor might be compromised by the pronouncement of death while there was still a heartbeat.

In 1968 we liberalized our criteria in accordance with the concept of irreversible brain injury as it was first outlined and applied at the University of Louvain, Belgium, by Alexandre and later defended by the Harvard ad hoc committee. Our experience since then has convinced us that anxieties about terminal care were unfounded. Acceptance of the brain death concept alleviated one of the most serious problems in liver transplantation, for it virtually eliminated the interval of normothermic ischemic injury and permitted the organ to be taken in the presence of an intact and effective circulation.

The subsequent preservation of the liver is also of vital importance and at our center we use one or more preservation modalities, depending on circumstances and always including organ hypothermia. With the acceptance of brain death as a criterion, it is often possible to maintain a naturally perfused liver in situ practically up to the moment of its excision. After removal, the liver may be quick-cooled by running a chilled electrolyte solution through the portal vein, thus lowering its temperature to about $10^{\circ}$ or $15^{\circ} \mathrm{C}$, which is sufficient for adequate preservation during the hour or so required for the vascular anastomoses in the 
recipient. Should the donor's heart stop before the recipient is ready, it is possible to employ the procedure used before 1968 (when cardiac arrest was required); by means of a heart-lung machine, circulation in the cadaver is reinstituted in combination with cooling. When longer periods of preservation and storage are needed (which has not been the case at this center since 1968) the liver may be removed and placed in a chamber such as that devised by Brettschneider, which combines perfusion, refrigeration, and hyperbaric oxygenation - this last process having an empiric favorable effect for reasons not clear. Conceivably, one could add to the perfusate a variety of metabolic inhibitors such as those reported by Webb, Fonkelsrud, and others. In one of our cases, the perfusion chamber permitted preservation of a liver without undue damage for nine hours, long enough for the recipient to make a transcontinental flight to Denver and to be prepared for surgery.

The above methods are still a far cry from the objectives of organ banking, and it must be conceded that there has been less progress in this than in any other aspect of transplantation. The importance of effective organ storage over the long term is obvious not only for reasons already noted but also to make it possible by portable devices to bring suitable and available organs to patients on a national exchange basis. Fresh ideas about organ preservation, either with solid state or perfusion techniques, are badly needed, for existing protocols seem to have reached the limit of their effectiveness. The importance of this problem has been acknowledged by a one-day "think tank" recently sponsored in Bethesda by the NIH, the Veterans Administration, and the National Research Council to smoke out some innovative ideas as well as to promote the funding with which to test these ideas.

Now, let us consider a few interesting aspects of the liver replacement operation. This procedure was first attempted in dogs by Francis Moore of Boston, and independently in our laboratories a short time later. As might be expected, the transition from animal experimentation to clinical application required some major technical adjustments and at least in one important and unexpected way demonstrated the need to be alert to the special requirements of human physiology. With removal of the host liver it is necessary to crossclamp temporarily the great veins draining the intestines and the lower half of the body. In dogs, if provision is not made for decompression of the distal venous pools during the anhepatic phase, the animals either die of shock on the operating table or expire at a later time because of irreparable damage to the mesenteric vessels. It was assumed that the same precaution would be necessary in humans and this was accomplished in the first five human recipients by plastic tube bypasses from splenic and/or femoral veins to the external jugulars. There was a dismaying incidence of pulmonary emboli that caused or contributed to the death of at least two of the recipients. It was suspected that the clots either originated within the bypasses and were actually carried to the lungs during the operation or were formed a short time later at or near the site where the femoral catheter had been inserted. To our relief, the omission of the venous decompression procedure in later patients did not produce any serious or long-lasting circulatory effects, including hypotension. Although a slight duskiness of the intestine developed in some recipients, it immediately disappeared when blood flow was restored through the reconstructed venous channels. One can explain the ease with which portal and vena caval cross-clamping was tolerated by man's inherently richer network of potential collateral channels for return of blood to the right heart.

Presumably, also, the size and ramifications of these vessels are further increased as a result of the disease in the liver. Venous decompression with bypasses has not been used in any recent case. The ability to omit this step has been a major technical advantage.

In planning a liver transplantation, the surgeon must be prepared for a high incidence of anatomic variations of either the graft or host structures. These have been noted in almost $40 \%$ of our cases. Multiple arteries have been the most frequent anomalies. When the recipient has had these, we have usually connected the graft celiac axis to the host aorta. 
When the multiplicity has been of the transprant vessels, multiple arterial anastomoses or other variant procedures have been used. There is no question that the need to improvise in these situations imposes an extra risk, particularly in very young recipients whose arteries are quite small and thin-walled. In patients with biliary atresia, anomalies may be so complex as to make it virtually impossible to succeed. Two of our infants with biliary atresia had a curious type of malrotation in which the portal vein passed in front of the pancreas and duodenum, the arterial blood supply issuing from the superior mesenteric artery rather than from the celiac axis, which was absent as was the retrohepatic inferior vena cava. In both cases corrective maneuvers to overcome the structural deficits were unsuccessful and the patients died within a few days.

The critical problem of obtaining adequate bile drainage and avoiding technical errors that may lead to leakage or obstruction may also be complicated by biliary tract anomalies, and the surgeon must be prepared to tailor his procedures to the individual case. An end-to-end anastomosis of the common duct, if it were normal, would have the advantage of preserving the sphincter of Oddi, thus providing drainage through a normal distal channel and reducing the chances of reflux of food or bacteria. This method, which of course is not available in the case of biliary atresia, is considered the procedure of choice by some surgeons. However, from our experience this anastomosis involves too high a risk of leakage and lnfection. We consider it undesirable to leave a T-tube prosthesis and a drain in an immunosuppressed patient when the duct reconstruction is in close proximity to the portal venous and hepatic arterial anastomoses. Further, there may not always be an adequate blood supply to the distal portion of the homograft common duct, which normally receives its principal arterialization from retroduodenal sources but now must depend on retrograde flow from arteries in the central hepatic hilum. Therefore, when feasible, we use the safer if somewhat less elegant technique of anastomosing the gallbladder directly to the duodenum and ligating the common duct.

Some dangers attend ligation of the transplant common duct if certain anomalies go unrecognized. Communication between the cystic and common ducts may not always be at the point of their apparent juncture. In one patient the ducts were externally fused but separated by an internal septum; in another, the homograft cystic duct passed behind the common duct and descended for almost two inches as one compartment of a double-barreled lumen. In both cases biliary drainage was inadvertently obstructed when the common duct ligature closed both parallel passages, a technical error subsequent surgery failed to correct and that proved fatal.

Some of the vascular and ductal anomalies could have been predicted, resulting either in better planning for surgery or in a decision not to operate at all. These earlier cases did not, however, have the benefit of the extensive arteriography and cholangiography that we now use routinely in the donor and sometimes in the recipient as well.

Other problems during and after operation may be caused by derangements in the coagulation mechanism that may result either in hemorrhage or thrombosis. As one would expect, acute bleeding can be particularly troublesome during the actual liver transplantation. To begin with, the very nature of the underlying hepatic pathology produces a portal hypertension in nearly every patient and the mechanics of the operation tend to exaggerate it. The usual consequence is mechanical bleeding that can rapidly assume nightmare proportions during the procedure. Many normal coagylation factors that might help control this unpleasant situation are dependent on the liver and are therefore defective in the diseased recipient. These coagulation factors may be even more deficient during the anhepatic phase, or they may subsequently be of dubious quality, depending on the state of 
preservation of the homograft - how much ischemia it has suffered and how much immediate functional capability it has retained.

When hemorrhage occurs, the surgeon must use any and all available hemostatic tactics ligating, suturing, cauterizing - until the revascularized homograft can participate in what is hoped will be appropriate coagulation function. With our earlier patients, whose homografts were generally of less than optimal quality for the reasons previously stated, we attempted to meet bleeding problems by administering thrombogenic agents. However,

hypercoagulability was caused in some instances. The unacceptable incidence of pulmonary embolism in these patients led us to abandon this approach.

In retrospect, it is possible that the coagulability induced by exogenous thrombogenic agents might be prohibitively additive to the clotting brought by the homograft, which, when it begins to function, conceivably overreacts. Indeed, the better the condition of the transplant, the greater the risk of unwanted coagulation. Almost every series of liver transplants, including our own, has at least one example of thrombosis of the hepatic arterial circulation to which the rebound phenomenon may have contributed. The use of anticoagulants to forestall this emergency is dangerous. Proved intravascular clotting during the operation would be an indication for heparin, but such proof is hard to come by. Moreover, heparinization is a double-edged maneuver: depressed clotting can have devastating effects on patients submitted to such major trauma and with so many potential bleeding sites.

In general, we now believe that it is advisable to avoid the manipulation of the clotting process either with thrombogenic or anticoagulant agents. Instead, our current approach is to leave correction of coagulation abnormalities to natural processes, intervening only under special circumstances and for very specific indications.

During operation, there are other metabolic abnormalities than those concerned with coagulation. Perhaps anesthesia deserves a comment here; it, too, is a complex problem in liver transplantation. Not only is the procedure long and difficult but, even more important, it is an operation on the very organ involved most in the metabolism and detoxification of most common anesthetics. At any point during the operation the liver is either inherently impaired, absent, or untried in its new setting. Hence the task in anesthesia is to administer correctly drugs that, first, are not hepatotoxic and, second, do not depend primarily on the liver for their degradation. The anesthesia in most of our early cases was administered by J. Antonio Aldrete. He has tended to rely mainly on such volatile agents as fluroxene added to a nitrous oxide-oxygen mixture in nonexplosive concentrations below $4.4 \%$. This combination permits use of electrocautery, gives flexibility in lightening or deepening anesthesia, and allows anesthesia to be abruptly stopped if the changing physiologic circumstances require it.

So much for some of the more important technical difficulties associated with liver transplantation. There is, of course, a long list of other technical pitfalls: adrenal venous infarction, air embolism, crushing of the right phrenic nerve by too high a clamp on the upper vena caval cuff, to mention but a few. The reader interested in a more detailed discussion of these and other surgical problems is referred to Experience in Hepatic Transplantation (see Selected Reading on page 60). These technical matters have played a major role in the mortality encountered in our first cases. However, as deaths from such causes are highly avoidable, surgical technique is of less critical significance to the future of transplantation than are the immunologic problems, The most important factor in successful transplantation remains the prevention of graft rejection by the host.

Immunosuppressive therapy in liver transplantation has borrowed heavil from the experience gained with human renal transplants. Two general treatment programs were 
evolved with the simpler kidney model and then applied to the liver recipients. The first protocol that was used from 1962 to 1966 for all organ recipients at the University of Colorado consisted of double-drug treatment with azathioprine and the synthetic adrenal cortical steroid, prednisone. Evolution of the use of these two agents together, appreciation of their marked synergism, and demonstration that rejection could be readily reversed by increasing the steroid doses were among the advances that made clinical transplantation practical and introduced what is known as the modern era of this field. But in spite moderately satisfactory results with renal transplantation, the double-drug therapy either did not prevent rejection of hepatic homografts or else proved too toxic to permit host survivaL Six patients so treated in liver transplantation from 1963 to 1965 died in a month or less.

In 1966, heterologous antilymphocyte globulin (A AG) was introduce clinically at our center as a third immunosuppressive agent, added to the drugs mentioned above. Since then this triple-drug therapy has been given to all our renal, hepatic, and cardiac recipients. In our experience, rejection has been more easily and regularly controlled, and the risks and morbidity imposed by the necessity for high-dose steroid therapy have been reduced. It must be admitted that not all transplant surgeons concede the need for ALG. However, those most familiar with its use have enthusiastically confirmed the value of the triple-drug regimen, beginning with Traejer of Lyon, France, and including most recently Simmons and Najarian of Minneapolis. The latter authorities advocate raising the heterologous serum with cultured lymphoblasts as the antigen rather than mature lymphoid tissue and recommend administering the subsequently refined ${ }_{A L G}$ by the intravenous rather than the originally employed intramuscular route: All of our human liver recipients who achieved chronic survival were treated with the combination of azathioprine, prednisone, and intramuscular AL G. In the event of a rejection episode, it is the steroid component that has proved most amenable to quick adjustment of dosage.

We will not review here the various hypotheses explaining the actions of these immunosuppressive drugs (see "A ntilymphocytic Serum : Its Properties and Potential," hospttal practice, May 1969. But, as was stated earlier, the method by which these agents are used in conjunction with the actual transplantation may permit selective abrogation of the host rejection response. Were this not true, there would be little hope of returning patients to life in an unrestricted environment since each of the individual agents can cause general immunologic crippling more or less in proportion to the dose used. The most obvious penalty of a depressed immune system is heightened susceptibility to infection. However, it has also become obvious that chronically immunosuppressed patients have an increased vulnerability to de novo malignancies. In our own series of chronic survivors after renal transplantation, more than 5\% have developed either mesenchymal or epithelial malignant tumors. Almost all other major transplantation centers have recorded this complication, which is presumably due to failure of the depressed immunologic surveillance mechanism to identify the tumor tissues as alien.

In addition to the foregoing general liabilities of immunosuppression, there are some special risks for the liver candidate. One is the fact that hepatic injury in all kinds of organ recipients has commonly been produced by the agents, individually or in combination, of the therapeutic regimen. In some instances, viral hepatitis, apparently made chronic by the partial immunologic invalidism of the host, has been a plausible explanation; but in others, hepatotoxicity of the drugs was probably responsible. With liver malfunction, dose control of some of the agents may become difficult since the liver participates in their pathways of action. These hepatic factors are obviously important in any situation requiring immunosuppression, but plainly they have heightened significance for a traumatized, transplanted liver fighting for survival in a new and hostile environment. 
Though infection is a major risk to any immunosuppressed patient, for the liver recipient postoperative sepsis of the graft itself has proved to be a special problem - partly because of the anatomic location of the orthotopically placed organ, interposed so to speak between the intestinal tract and the heart. Bacteria from the bowel, particularly of the gram-negative variety, can be brought into contact with the transplanted liver via intestinal veins draining into the portal vein, or, alternatively, by retrograde spread up the duct system after passage through the biliary anastomosis. In either event, the presence of nonviable hepatic tissue provides a perfect medium for bacterial growth. Eventually, piecemeal gangrene of the transplant can result, with characteristic nonvisualizing areas on the liver scans, gramnegative bacteremia, and all the findings of generalized sepsis.

Early in Our clinical series, the above findings of graft and systemic infection led us to consider the essential problem to be that of bacterial invasion and prompted us to reduce the immunosuppression. This decision was tragically incorrect and was followed by necrosis and infection of large parenchymal areas. Experience soon taught us that ischemia of portions of the liver was the initiating event, and that the basis for the ischemia was rejection. Consequently, immunosuppression should ordinarily be increased rather than reduced if this complication is thought to be impending. When this was done by giving substantially higher doses of prednisone (as noted, the only highly dose-maneuverable component of the immunosuppressive triad), the incidence of regional hepatic gangrene fell to zero. It should be added that our prophylactic treatment protocol includes heavy antibiotic treatment for the first postoperative week, including agents effective against gram-negative bacteria, after which this therapy is stopped.

With the acquisition of experience, other important issues have also been clarified, including that of the indications for liver replacement, A brief summary of our first 33 consecutive recipients, treated from March 1963 to 12 months ago, can be used to illustrate these indications.

The 33 patients were aged 11 months to 68 years. The indication for 12 of these transplants was hepatoma, and an additional unsuspected hepatoma was found in a four-year-old child treated for intrahepatic biliary atresia. The latter is the only one of this group still alive; now 12 months after the operation she shows no evidence of recurrence of neoplasia. As for the other hepatoma patients, seven died within 39 days. Badly damaged homografts were a major cause of failure ill five who were among our early patients in the period before the criterion of brain death was applied, while technical accidents, with subphrenic abscesses and bile peritonitis, were the major causes of death in the other two. Of the five hepatoma patients who had more prolonged survival $-76,143,339,400$, and 432 days-metastases developed in all, and in four instances the recurrences were directly responsible for death. Because of this high rate of recurrent carcinoma, it has become our policy to consider liver replacement for hepatoma only under the most exceptional circumstances, even though our experience and that of Caine have demonstrated the possibility of an occasional tumor cure.

Far more desirable candidates are those without neoplasms even though the technical difficulties in benign hepatic disease are more severe because the patients tend to be sicker and to have more advanced portal hypertension. Moreover, if the diagnosis is biliary atresia, an increased incidence of vascular anomalies can be expected to compound the difficulties together with the small size of the structures to be anastomosed in these young patients. Nevertheless, the longest survivors of liver transplantation in the world are those who had this disorder. Of our own series of 15 patients with biliary atresia, treated a year or longer ago, including the child with the incidental hepatoma mentioned above, five lived longer than one year and two are still surviving with completely normal liver function, one year and two and a half years, respectively, after operation. The three late deaths after 13, 131/2, and 
30 months were from recurrent hepatic insufficiency caused in two instances by chronic rejection and in the third probably by indolent viral hepatitis. Another four children survived 61 to 186 days, all expiring from regional hepatic gangrene for which the apparent cause was too little immunosuppression in the early posttransplantation period. The six other recipients with biliary atresia died within the first 40 days, two from hepatic necrosis probably attributable to ischemia of the donor organ, two from thrombosis of the hepatic artery or portal vein, one from a nonthrombotic occlusion of the hepatic artery, and the remaining one from generalized infection and pneumonia.

Six patients were treated for cirrhosis a year or longer ago, with the disappointing record of five early deaths. Reasons for the heavy acute mortality included the wretched physical condition of the recipients, major hemorrhage due to portal hypertension and depressed coagulation, and technical or metabolic mishaps. The survivor is an 11-year-old child whose cirrhosis was secondary to Wilson's disease. After liver replacement the child suffered an extremely severe rejection crisis, but the process was eventually reversed, with normal liver function now continuing 18 months after the operation. Moreover, the new liver, which was biopsied at 6 and 17 months, has not reaccumulated copper and it appears to have corrected the genetic error in copper metabolism - a finding that may be important in the search for the etiology of this disease.

Undoubtedly, one reason for the bad experience with cirrhotic patients has been a reluctance to recommend such therapy except in the agonal stages of the disease. But now that the feasibility of long-term survival and rehabilitation has been demonstrated, transplantation at an earlier time probably should be considered, particularly in postnecrotic cirrhosis in which the maximum value of medical management and of abstinence from alcohol has already been realized.

With another of our patients - one not in the series described above - there have been exceptionally interesting circumstances of transplantation. A 28-year-old woman with chronic active hepatitis, she was operated on August 9, 1970. For at least two years before the operation, as well as on the day of the transplantation, immunodiffusion tests for the Australia antigen were positive. This gave us an opportunity to examine the thesis that this antigen has an essentially hepatic source. The argument for this proposition was supported by the immediate disappearance of the antigen from the bloodstream after the operation. Almost two months later it reappeared, and within a few days there followed an attack of acute serum hepatitis with joint pain, anorexia, jaundice, and evidence of hepatic necrosis by transaminase determinations. Fortunately, her liver function abnormalities have regressed nearly completely, but she is still Australia antigen-positive, now five months posttransplantation. It remains to be seen whether the virus will doom the new liver to the same fate as the old one, whether the long-term pace of the infection will be affected by the chronic immunosuppression, and whether the patient will continue to be a virus carrier. The case has other interesting implications. It is customary to think of the incubation period of infectious diseases in terms of the host immune defenses. In this patient, the immune apparatus was retained but systematically weakened. Yet, the latent period of the disease was about that to be expected with a fresh infection, indicating that the incubation period in this patient was primarily concerned with the target organ.

Thus far, I have confined discussion strictly to the kind of operation (termed orthotopic transplantation) in which the diseased host liver is removed and replaced. The alternative to this procedure in patients with benign disease is auxiliary hepatic transplantation, in which the native liver is not disturbed and the hepatic homograft is placed in some abnormal location such as the paravertebral gutter, splenic fossa, or pelvis. Special technical and metabolic problems that have been encountered with auxiliary transplantation cannot be 
reviewed here but they have been detailed in Experience in Hepatic Transplantation. The results with the auxiliary procedure in animals and in limited clinical trials have not been particularly encouraging.

In summary, orthotopic liver transplantation has led to the prolongation of useful life. Among our first 33 recipients, 9 lived a year or longer, and their chances for survival rose with the increase in our experience. The longest survival to date exceeds $2 \frac{1}{2} 2$ years. With more experience, with successful efforts to reduce the toxicity of immunosuppressive agents, with improvements in histocompatibility typing, and with advances in preservation and storage techniques, the record can be expected to improve. Even now, patients with nonmalignant terminal liver disease and with no other hope for recovery can be candidates for liver transplantation.

\section{Selected Reading}

1. Liver Transplantation. Moller G. Transplant Rev. 1969; 2:3-68. (The entire issue of this journal is concerned with liver transplantation. Among other articles, it contains accounts by R. Calne, A. C. Birch, and F. D. Moore about the clinical experience with liver transplantntion at Cambridge and Harvard universities.). [PubMed: 4333563]

2. Fortner JG, Beattie EJ, Shiu MH, Kawano N, Howland WS. Orthotopic and heterotopic liver homografts in man. Ann Surg. 1970; 172:23-32. [PubMed: 4913058]

3. Starzl, TE. Experience in Renal Transplantation. WB Snunders Company; 1964.

4. Starzl, TE.; Putnam, CW. Experience in Hepatic Transplantation. WB Saunders Company; 1969. 


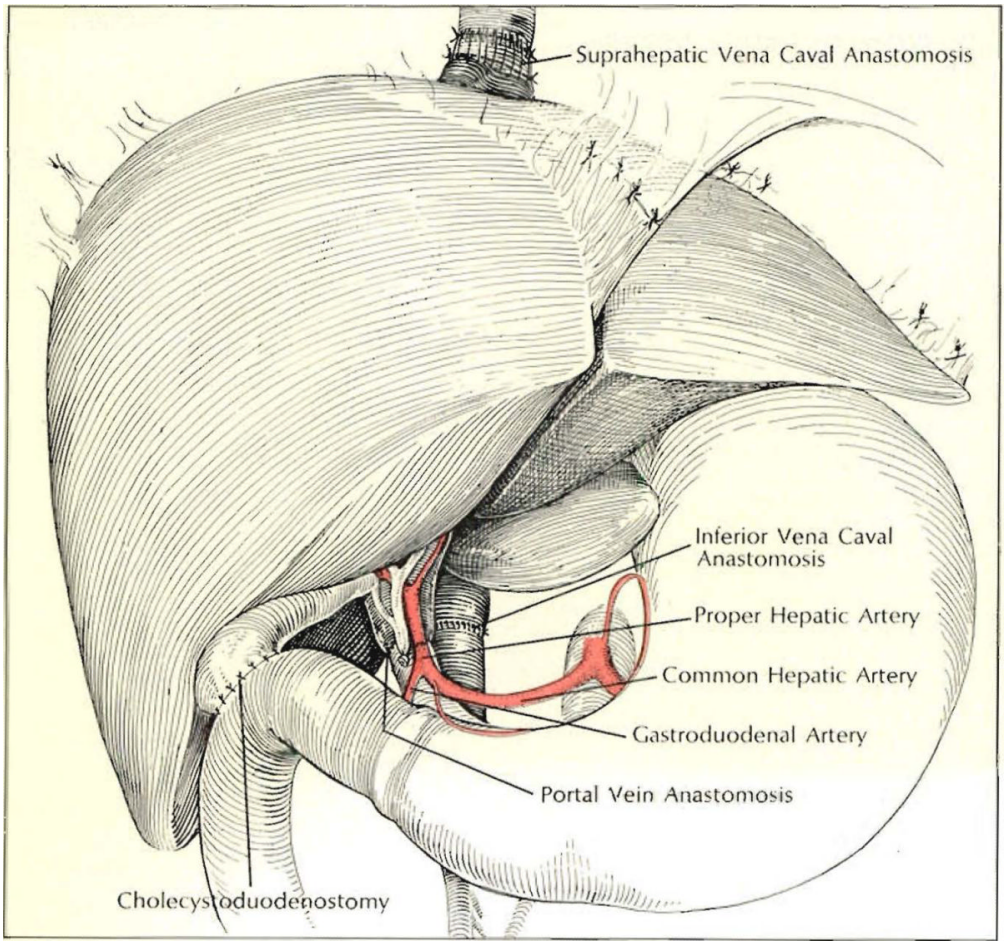

1.. Insertion of second homograft in child pictured opposite proceeded as shown above, with cholecystoduodenostomy performed for biliary drainage and the donoor celiac axis attached to the recipient proper hepatic artery. Note that a cuff of the first homograft's inferior vena cava was left to lengthen the suprahepatic connection. 


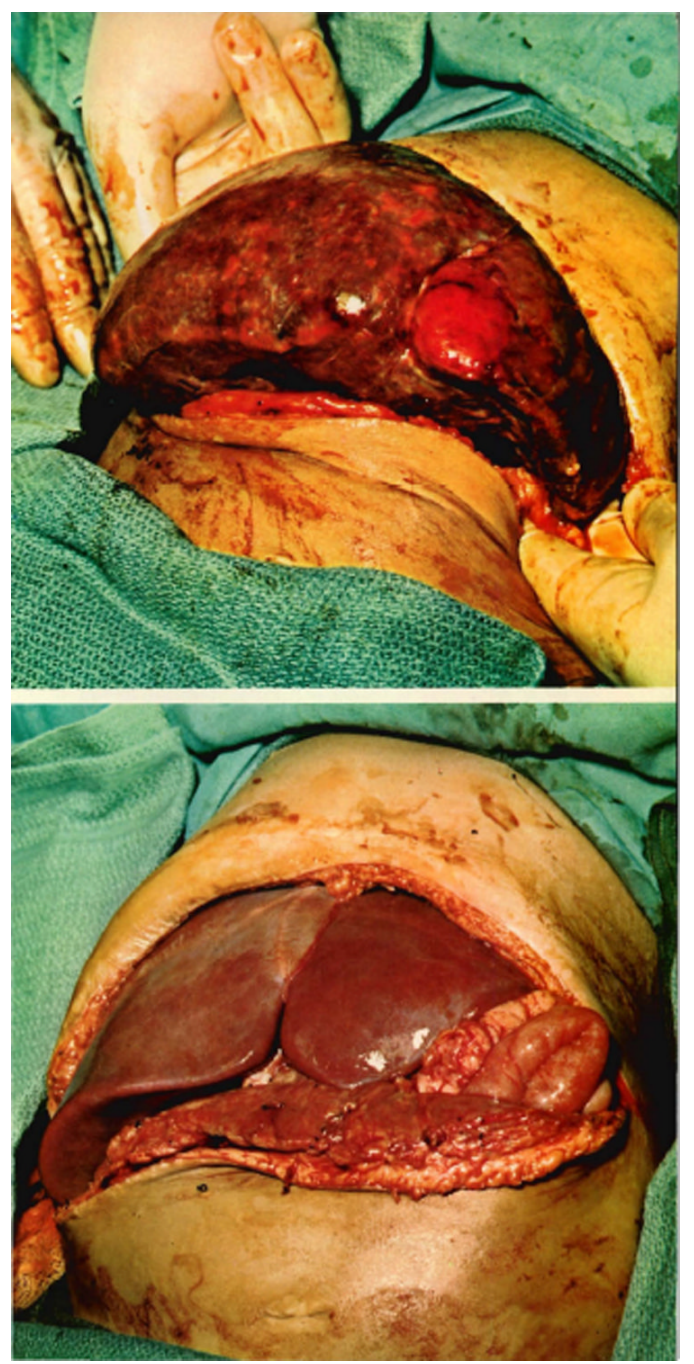

2. .

A rejecting homograft in a two-year-old child treated by transplantation for extra-hepatic biliary atresia is shown at top at the time of its removal, 68 days following insertion. (The liver weighed $250 \mathrm{gm}$ at insertion, 880 at removal.) The child received a second homograft (lower photo); his subsequent course was extremely complicated, in part because of the large size of the second liver (which came from a seven-year-old donor), which severely crowded the abdominal cavity. Fortunately, swelling due to rejection was delayed for a month and eventually the child was able to eat a full diet. Although jaundiced, the child lived for one year after the second transplant and died of hepatic failure. (For gross appearance of first graft on pathologic examination see next page.) 


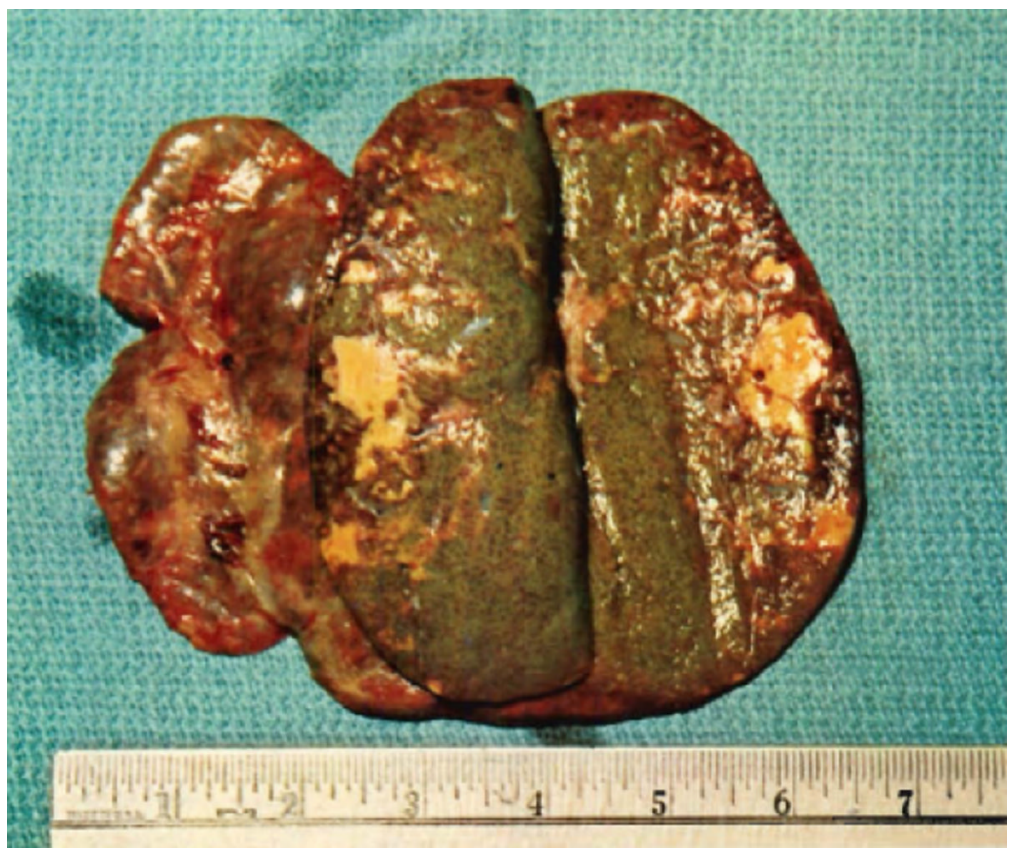

3. . Gross subcortical infarcts were easily seen in the rejected homograft removed from the patient described on pages 48-49. Some of the necrotic areas revealed hy slicing appeared to have been devitalized very recently, others seemed to be older, and both types tended to be near the surface. The major blood vessels were found free of clots. 

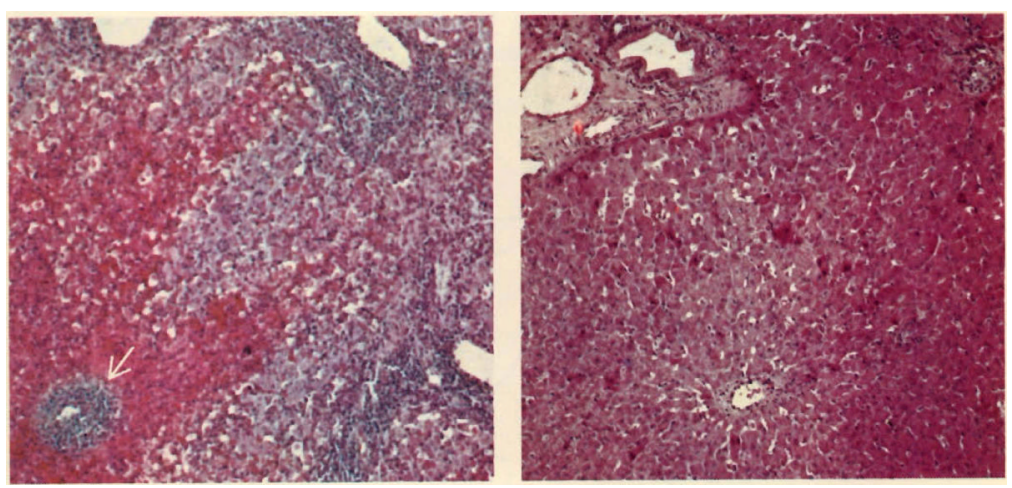

4. .

An untreated canine hepatic homograft is shown six days after transplantation (left). Portal veins (clear spaces) and central vein (arrow) are surrounded by dense cellular infiltration and there is centrilobular necrosis with hemorrhage (hematoxylin and eosin stain, $\times 30$ ). Photo at right shows normal liver architecture in another canine homograft one year after transplantation. This dog received azathioprine for four months, no additional therapy. It is still alive after some seven years. 


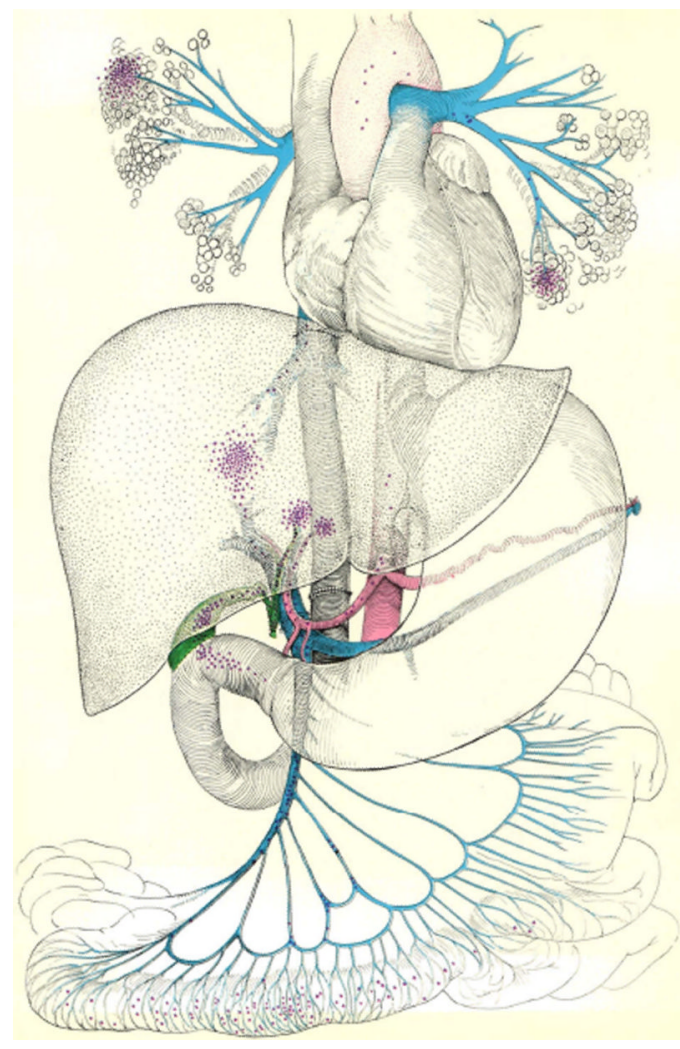

5.

The location of the orthotopic liver homograft, between the bowel and the heart, helps explain the predisposition of these patients to graft sepsis and eventual gangrene. As the drawing opposite suggests, invading microorganisms (purple dots) may enter via portal vein or through the recollstructed biliary tract; the lungs also are at high risk. 

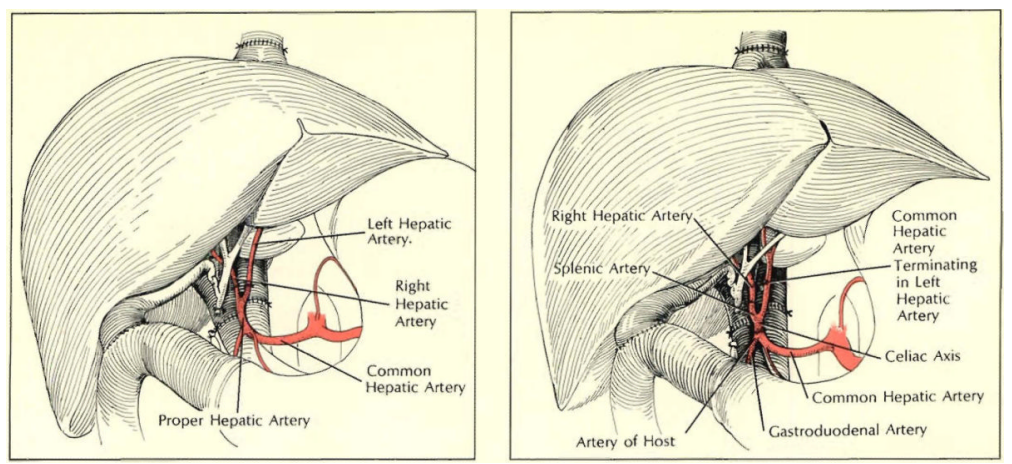

6. .

Multiple arteries, in either graft or host, often complicate operation. In both situations shown, two graft arteries were encountered. Left: problem was solved by individually anastomosing the vessels to branches of recipient hepatic artery. Right: donor celiac axis was first joined to recipient common hepatic artery. After blood flow was restored through larger left branch, other (right) donor artery, which had originated from superior mesenteric artery, was revascularized by being attached to donor splenic artery. 

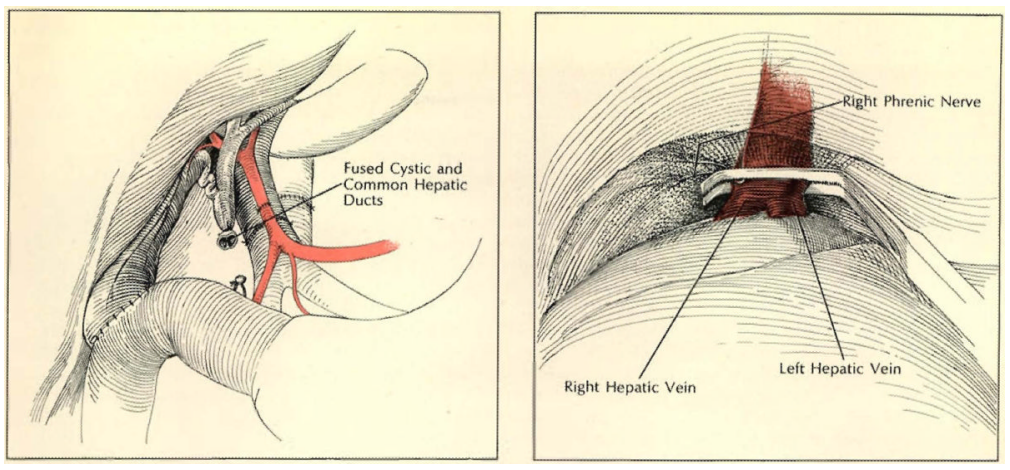

7. .

Anomalies of communication between the cystic and common ducts must also be watched for during operation. In situation shown at left, the cystic and common ducts were externally fused but separated by all internal septum. When cholecystoduodenostomy and common duct ligation were carried out, the result was total biliary obstruction. At right, right phrenic nerve is inadvertently included in clamp occluding suprahepatic vena cava, as is a piece of the diaphragm. Temporary phrenic paralysis resulted. 


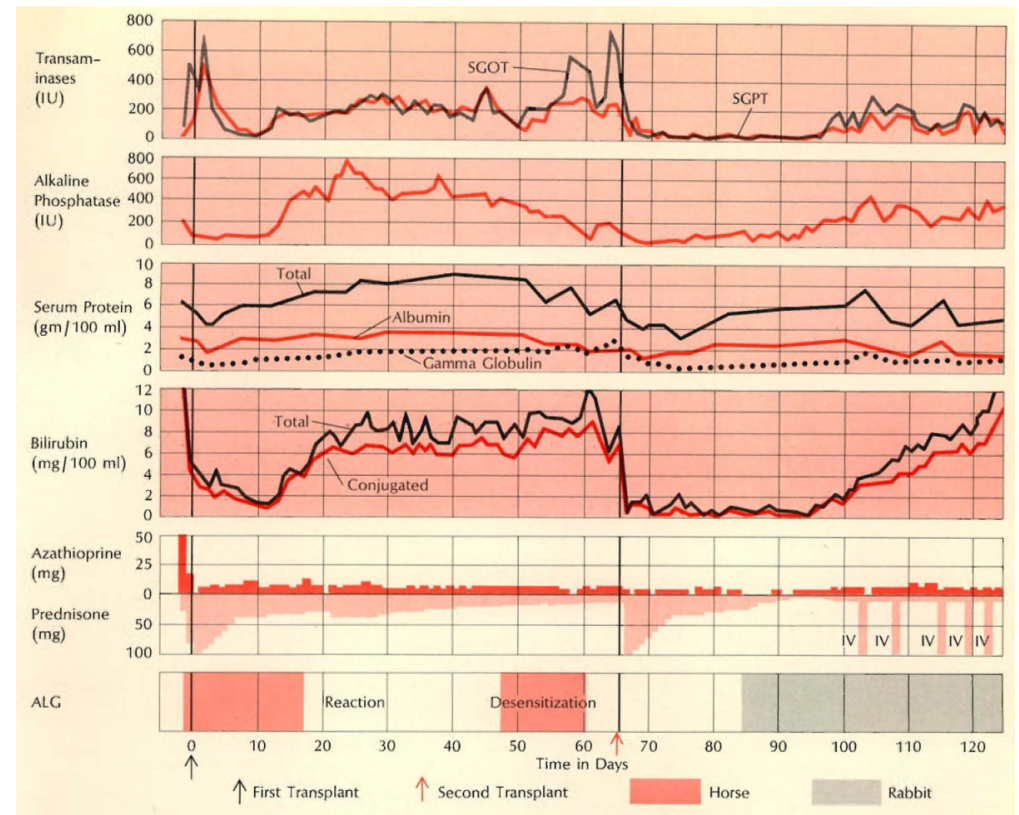

8. .

An "indolent" type of rejection characterized the clinical course of the child who received two homografts (see page 49); this proved to be the type most difficult to treat. Rejection of the first transplant began on the 13th postoperative day and progressed slowly; horse ALG had to be stopped after 18 days because of severe local reaction, Jaundice then developed; other measures of liver function were maintained for many weeks despite the fact that the child was extremely ill. Desensitization was attempted in the hope of resuming horse ALG treatment but was eventually abandoned. Note hypergammaglobulinemia toward end of second month. Onset of rejection after second transplant was delayed about a month; by this time rabbit ALG was available for treatment. 

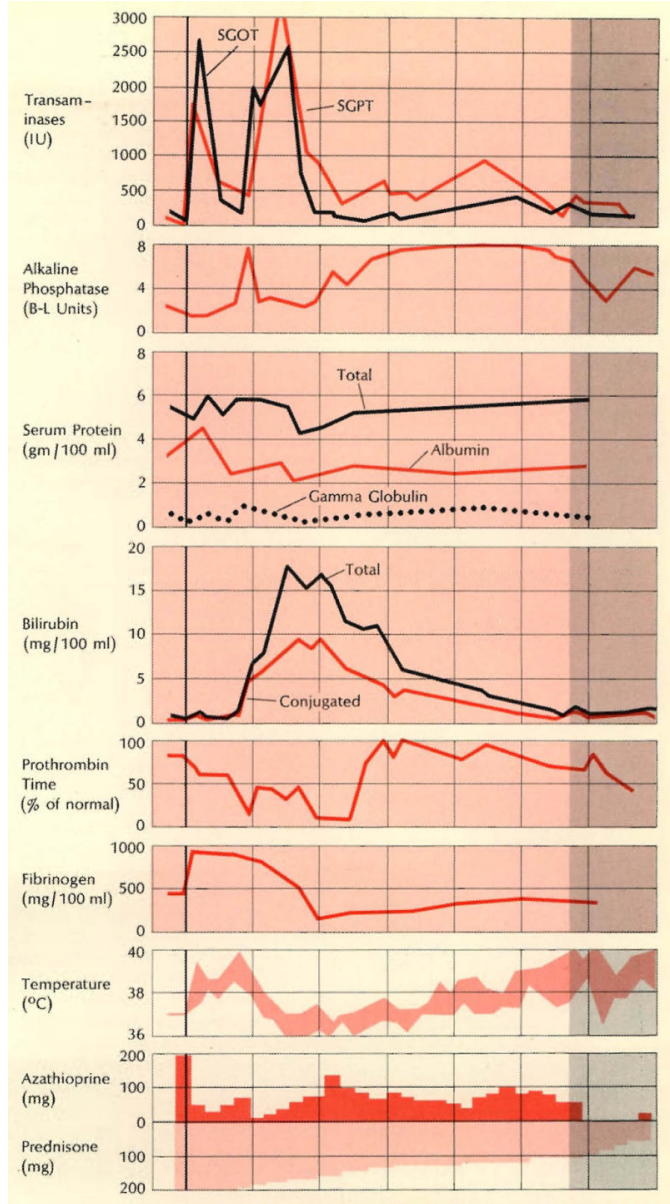

$(\mathrm{mg})$

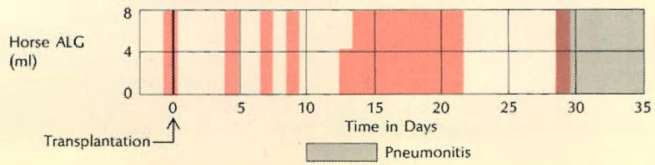

9. .

The course of an acute rejection is shown at right in record of a 23-year-old woman who underwent transplantation for hepatoma. Although the rejection crisis was overcome promptly, she died of pseudomonas pneumonia after 35 days. Even before the onset of jaundice her course was markedly febrile and she had severe bleeding problems: note marked depression of prothrombin time and fibrinogen concentration on days 10 through 13. 


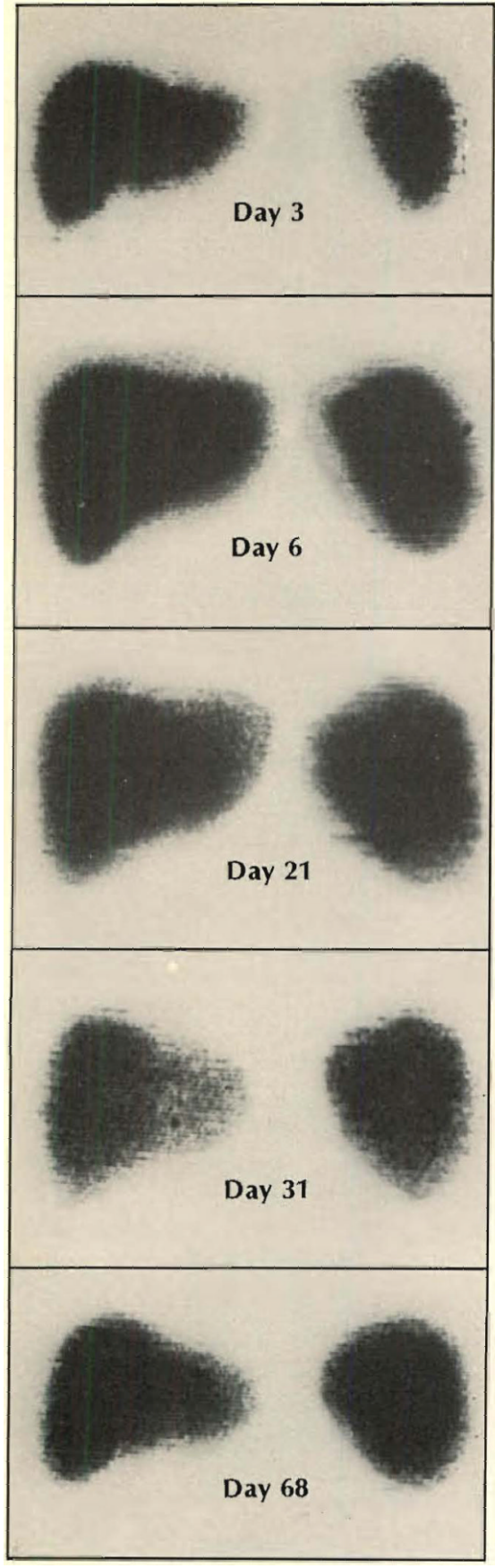

10. .

Scans using TC $99 \mathrm{M}$ were made in 15-year-old boy treated for hepatoma. Rejection began about a week after operation, as swelling on day 6 indicates, and lasted about two weeks. By day 68 anteroposterior scan returned to about the same dimensions as had heen present shortly after the transplantation, but the lateral view showed that the liver mass was increased. Liver function at this time was completely normal. The pickup of the isotope remained homogeneous through th e period of observation, except possibly at 31 days. The homograft was eventually destroyed by tumor recurrence and the boy died 143 days after the transplant operation. 

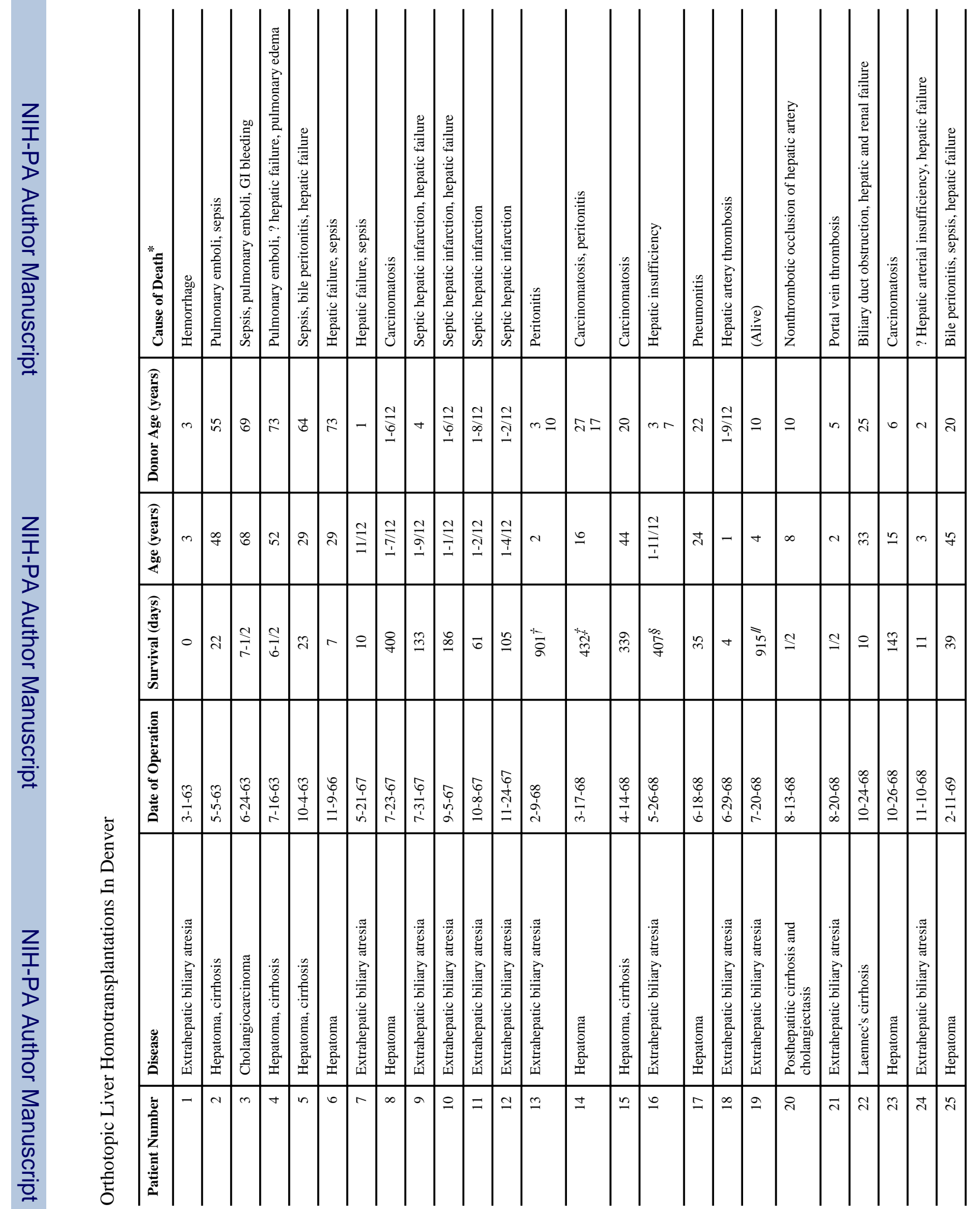
STARZL

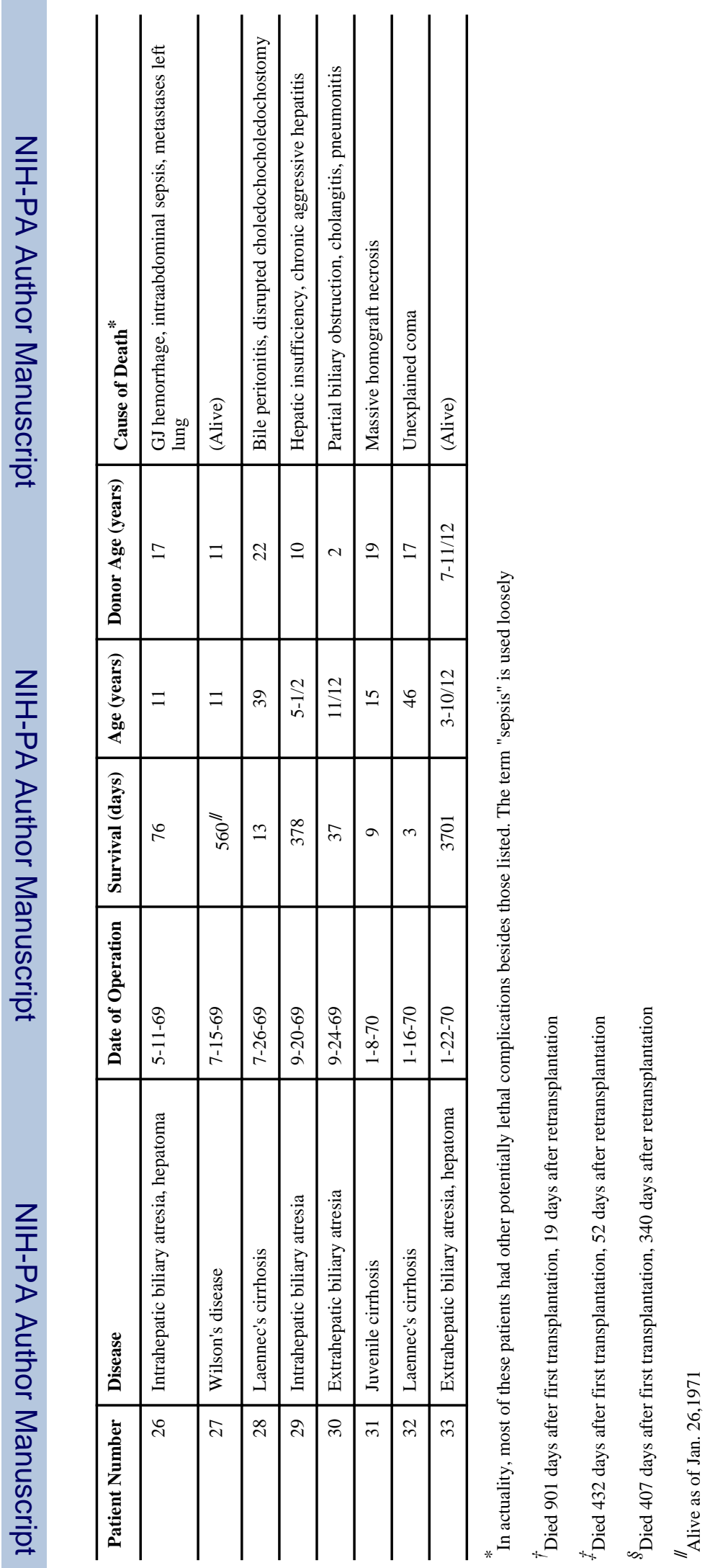

Hosp Pract (Hosp Ed). Author manuscript; available in PMC 2011 May 3. 